\section{Defective AP-3-dependent VAMP8 trafficking impairs Weibel-Palade body exocytosis in Hermansky-Pudlak Syndrome type 2 blood outgrowth endothelial cells}

Ellie Karampini, ${ }^{1,{ }^{\star}}$ Maaike Schillemans, ${ }^{1, *}$ Menno Hofman, ${ }^{1}$ Floris van Alphen, ${ }^{2}$ Martin de Boer, ${ }^{3}$ Taco W. Kuijpers, ${ }^{3,4}$ Maartje van den Biggelaar, ${ }^{1}$ Jan Voorberg ${ }^{1,5}$ and Ruben Bierings ${ }^{1,6}$

\begin{abstract}
${ }^{1}$ Molecular and Cellular Hemostasis, Sanquin Research and Landsteiner Laboratory, Amsterdam UMC, University of Amsterdam, Amsterdam; ${ }^{2}$ Research Facilities, Sanquin Research and Landsteiner Laboratory, Amsterdam UMC, University of Amsterdam, Amsterdam; ${ }^{3}$ Blood Cell Research, Sanquin Research and Landsteiner Laboratory, Amsterdam UMC, University of Amsterdam, Amsterdam; ${ }^{4}$ Pediatric Hematology, Immunology and Infectious Disease, Amsterdam UMC, University of Amsterdam, Amsterdam; ${ }^{5}$ Experimental Vascular Medicine, Amsterdam UMC, University of Amsterdam, Amsterdam and ${ }^{6} \mathrm{Hematology}$, Erasmus University Medical Center, Rotterdam, the Netherlands

${ }^{*} E K$ and MS contributed equally to this work.
\end{abstract}

\section{ABSTRACT}

W eibel-Palade bodies are endothelial secretory organelles that contain von Willebrand factor, P-selectin and CD63. Release of von Willebrand factor from Weibel-Palade bodies is crucial for platelet adhesion during primary hemostasis. Endosomal trafficking of proteins like CD63 to Weibel-Palade bodies during maturation is dependent on the adaptor protein complex 3 complex. Mutations in the AP3B1 gene, which encodes the adaptor protein complex $3 \beta 1$ subunit, result in HermanskyPudlak syndrome 2 , a rare genetic disorder that leads to neutropenia and a mild bleeding diathesis. This is caused by abnormal granule formation in neutrophils and platelets due to defects in trafficking of cargo to secretory organelles. The impact of these defects on the secretory pathway of the endothelium is largely unknown. In this study, we investigated the role of adaptor protein complex 3-dependent mechanisms in trafficking of proteins during Weibel-Palade body maturation in endothelial cells. An ex vivo patient-derived endothelial model of Hermansky-Pudlak syndrome type 2 was established using blood outgrowth endothelial cells that were isolated from a patient with compound heterozygous mutations in AP3B1. Hermansky-Pudlak syndrome type 2 endothelial cells and CRISPR-Cas9engineered $A P 3 B 1 \%$ endothelial cells contain Weibel-Palade bodies that are entirely devoid of CD63, indicative of disrupted endosomal trafficking. Hermansky-Pudlak syndrome type 2 endothelial cells have impaired $\mathrm{Ca} 2^{+}$mediated and cAMP-mediated exocytosis. Whole proteome analysis revealed that, apart from adaptor protein complex $3 \beta 1$, also the $\mu 1$ subunit and the v-SNARE VAMP8 were depleted. Stimulus-induced von Willebrand factor secretion was impaired in CRISPR-Cas9-engineered VAMP8endothelial cells. Our data show that defects in adaptor protein complex 3dependent maturation of Weibel-Palade bodies impairs exocytosis by affecting the recruitment of VAMP8.

\section{Introduction}

Weibel-Palade bodies (WPB) are the storage and secretory compartment of endothelial cells and play an important role in hemostasis, inflammation and angiogenesis. ${ }^{1}$ Secretion of their main cargo, the hemostatic protein von Willebrand factor (vWF), promotes platelet adhesion at the site of injury. ${ }^{2}$ Apart
Haematologica 2019

Volume 104(10):2091-2099

\section{Correspondence:}

RUBEN BIERINGS

r.bierings@erasmusmc.nl

Received: October 7, 2018.

Accepted: January 9, 2019.

Pre-published: January 10, 2019.

doi:10.3324/haematol.2018.207787

Check the online version for the most updated information on this article, online supplements, and information on authorship \& disclosures: www.haematologica.org/content/104/10/2091

(C)2019 Ferrata Storti Foundation

Material published in Haematologica is covered by copyright. All rights are reserved to the Ferrata Storti Foundation. Use of published material is allowed under the following terms and conditions:

https://creativecommons.org/licenses/by-nc/4.0/legalcode. Copies of published material are allowed for personal or internal use. Sharing published material for non-commercial purposes is subject to the following conditions: https://creativecommons. org//icenses/by-nc/4.0/legalcode sect. 3. Reproducing and sharing published material for commercial purposes is not allowed without permission in writing from the publisher. 
from vWF, WPB also store angiopoietin-2, IGFBP7 and various chemokines, along with the transmembrane protein $\mathrm{P}$-selectin and the integral membrane protein CD63. ${ }^{1,3}$ Simultaneous release of this cocktail of inflammatory and angiogenic mediators from WPB also promotes extravasation of leukocytes and vessel repair mechanisms. Weibel-Palade bodies belong to the lysosome-related organelles (LRO), a heterogeneous group of subcellular organelles that share features with lysosomes through acquisition of recycled cargo and/or membrane components from the endo-lysosomal system. ${ }^{4}$ Biogenesis and subsequent degranulation of LRO is fundamental to the function of a wide variety of (circulating) cells, including granulocytes, $\mathrm{T}$ cells, platelets and endothelial cells. Although their function and cargo differ between cell types, the mechanisms and core components that control LRO biogenesis, maturation and degranulation are shared and operate in all cells with LRO. In endothelial cells, biogenesis of WPB starts at the trans-Golgi Network (TGN) and is driven by the biosynthesis of vWF. At this point, other soluble cargo, as well as P-selectin, are also included in newly forming WPB. In a subsequent post-Golgi step during WPB maturation, additional key components, such as CD63, are transferred from adaptor protein complex 3 (AP-3)-positive endosomes to maturing WPB. ${ }^{5-7} \mathrm{AP}-3$ is a heterotetrameric complex, consisting of four subunits: $\beta 1, \delta 1, \beta 1$ and $\sigma 1$, previously also referred to as $\beta 3 \mathrm{~A}-, \delta 3-, \mu 3 \mathrm{~A}$ - and $\sigma 3 \mathrm{~A}$ adaptins, respectively. ${ }^{8}$ The AP-3 $\mu 1$ subunit is known to interact with membrane proteins through linear sequences of amino acid residues in their cytoplasmic tail, such as the di-leucine ([DE]XXX[LI]) and the tyrosine (YXXØ) motifs, ${ }^{9,10}$ the latter of which is also present in CD63 (GYEVM). ${ }^{11}$ When its tyrosine motif is altered or the expression of AP-3 11 is down-regulated, CD63 shows impaired trafficking to WPB, suggestive of a direct interaction between the AP-3 complex and CD63.

Defective formation and degranulation of LRO is at the basis of a number of poorly understood congenital storage pool disorders (SPD) that affect secretory responses of cells. Since the mechanisms of LRO formation and degranulation are shared between different cell types, SPD are often polysystemic, affecting many cell types at the same time which leads to complex disease symptoms. Hermansky-Pudlak syndrome (HPS) is a group of autosomal recessive disorders characterized by hypopigmentation and platelet storage pool deficiency, due to defective maturation of melanosomes and platelet dense granules, respectively. ${ }^{12}$ HPS-2, a subtype of HPS, affects the expression and functionality of the AP-3 complex by mutations in the AP3B1 gene, which encodes the AP-3 complex $\beta 1$ subunit. ${ }^{13}$ Apart from the shared HPS features of platelet dysfunction and albinism, HPS-2 is also uniquely characterized by $\mathrm{CD} 8^{+}$cytotoxic T-cell dysfunction and neutropenia. ${ }^{14-16}$

Given the polysystemic nature of SPD, we sought to determine how genetic deficiencies in the AP-3 sorting machinery impact the secretory function of endothelial cells. Here we show, using blood outgrowth endothelial cells (BOEC) from an HPS-2 patient, that defects in AP-3 dependent maturation of WPB impairs the exocytotic potential of WPB by affecting the recruitment of the WPB-localized member of the SNARE fusion machinery VAMP8.

\section{Methods}

\section{Cell culture and isolation of blood outgrowth endothelial cells}

Blood outgrowth endothelial cells were isolated as previously described and cultured in EGM-2 (Lonza, Basel, Switzerland, CC-3162) supplemented with $18 \%$ fetal calf serum (FCS) (Bodinco, Alkmaar, the Netherlands) (EGM-18). ${ }^{17}$ HPS-2 BOEC were isolated from venous blood from a patient diagnosed with HPS-2 (described by de Boer et al. ${ }^{16}$ ), caused by compound heterozygote AP3B1 mutations (c.177delA, p.K59Nfs*4 and c.18391842delTAGA, p.D613Efs*38). The study was performed according to national regulations regarding the use of human materials. The patient's parents signed an informed consent form allowing participation. Control BOEC were isolated from healthy, anonimized donors participating in the voluntary internal blood donor system of Sanquin Blood Supply following written consent. The study was approved by the Medical Ethical Committee of the Academic Medical Center in Amsterdam and was conducted in accordance with the Declaration of Helsinki.

\section{DNA constructs}

The mEGFP-LIC and LVX-mEGFP-LIC vectors have been described before. ${ }^{18,19}$ The EGFP-AP-3 $\beta 1$ plasmid encoding AP-3 $\beta$ with EGFP fused to its aminoterminus was a gift from Dr. Adolfo Saiardi. ${ }^{20}$ To construct LVX-mEGFP-AP3- $\beta 1$, a 3317 bp fragment containing the AP-3 $\beta 1$ coding sequence was cut from EGFP-AP-3 $\beta 1$ using BsrGI and SacII and pasted in frame behind mEGFP using the same sites in mEGFP-LIC. In a second step, the AP-3 $\beta 1$ coding sequence was excised from mEGFP-AP-3 $\beta 1$ using BsrGI and NotI and pasted in frame behind mEGFP in the LVX-mEGFP-LIC vector.

\section{CRISPR genome engineering}

gRNA were designed to target exon 1 of the $A P 3 B 1$ gene and exon 1 and 2 of the VAMP8 gene using the CRISPR Design tool (http://crispr.mit.edu). gRNAs [(AP3B1 exon 1: gRNA-4: TACAATGAGCAGTCCGGAGG and gRNA-5: ACAATGAGCAGTCCG GAGGA); (VAMP8 exon 1: gRNA-4: GAATGTGGAGCGGATCCTGG and gRNA-5: AGA ATGTGGAGCGGATCCTG; exon 2: gRNA-3: CTGGAGCGACTCGAGATGCG)] were selected based on the specificity score with the minimum amount of off-target effects and were subsequently cloned as hybridized oligos [(AP3B1: gRNA-4: RBNL306 5'-CA CCGTACAATGAGCAGTCCGGAGG-3' and RBNL307 5'-AAACCCTCCGGACTGCTCA TTGTAC-3'; gRNA-5: RBNL308 5'CACCGACAATGAGCAGTCCGGAGGA-3' and RBNL309 5'AAACTCCTCCGGACTGCTCATTGT C-3'), (VAMP8: gRNA3: RBNL318 5'-CACCGGTGGAGGAAATGATCGTGTG-3' and RBNL319 5'-AAACCACACGATCATT TCCTCCACC-3'; gRNA-4: RBNL320 5'-CACCGATTCACTTACTGACCGGCCT-3' and RBNL321 5'-AAACAGGCCGGTCAGTAAGTGAATC-3'; gRNA-5: RBNL322 5'-CACCGA TTCACTTACTGACCGGCCT-3' and RBNL323 5'-AAACGGCCGGTCTCAGTAAGTGAA TTC-3')] into BsmBI-digested LentiCRISPR_v2 vector (a gift from Dr. Feng Zhang; Addgene \#52961). A detailed protocol on transduction and clonal selection of knockout BOEC has been previously described. ${ }^{21}$

\section{CD63 and CD62P membrane exposure}

Endothelial cells were cultured in gelatin-coated 6-well plates until confluency for 3-4 days prior to the experiment. In order to measure CD63 surface exposure in WT and HPS-2 BOEC under steady conditions, cells were washed twice with phosphate buffered saline (PBS), detached with Accutase (Sigma, A6964), 
and stained with FITC-conjugated anti-CD63 antibody at $4^{\circ} \mathrm{C}$ for 30 minutes ( $\mathrm{min}$ ) in the dark. After incubation, cells were washed with PBS, spun down at $250 \mathrm{xg}, 4^{\circ} \mathrm{C}$ for 2 min and suspended in PBS containing $0.01 \%(\mathrm{v} / \mathrm{v}) \mathrm{NaN} 3$. Samples were measured by flow cytometry (BD FACSCANTO II, BD Biosciences). In order to measure CD62P levels on the plasma membrane upon stimulation, cells were pre-incubated in release medium for at least $30 \mathrm{~min}$. Stimulation was performed in RM supplemented with $100 \mu \mathrm{M}$ histamine for $5 \mathrm{~min}$. After stimulation, cells were treated with ice-cold $\mathrm{PBS}$ and detached by Accutase. Staining for CD62P exposure was performed at $4^{\circ} \mathrm{C}$ in the dark with a PE-conjugated anti-CD62P antibody for $20 \mathrm{~min}$. Cells were centrifuged at $4^{\circ} \mathrm{C}$ at $250 \mathrm{xg}$ for $2 \mathrm{~min}$ and subsequently resuspended in $\mathrm{PBS}$ containing $0.01 \%(\mathrm{v} / \mathrm{v}) \mathrm{NaN}_{3}$.

Further details on materials and methods are available in the Online Supplementary Appendix.

\section{Results}

\section{AP3B1 deficient endothelial cells exhibit impaired intracellular protein trafficking from endosomes to Weibel-Palade bodies}

To study the role of the AP-3 complex in primary endothelial cells, we isolated BOEC from peripheral blood mononuclear cells of an HPS-2 patient with mutations in the AP3B1 gene that encodes for the $\beta 1$ subunit of the heterotetrameric AP-3 complex. The patient, who has previously been described by de Boer et al., ${ }^{16}$ is a compound heterozygote with both mutations (exon 2: c.177delA, p.K59Nfs4; exon 17: c.1839-1842delTAGA, p.D613Efs*38) leading to a frame shift and a premature stop codon (Figure 1A). As expected, western blot analysis of lysates of BOEC confirmed that the expressed levels of AP-3 $\beta 1$ were not detectable (Figure $1 \mathrm{~B}$ ). The truncated AP-3 $\beta 1$ protein products, generated in the HPS- 2 BOEC, are most likely rapidly degraded as previously reported in HPS-2 fibroblasts. ${ }^{13}$ In order to test whether there is any remaining functionality of the AP-3 complex in HPS-2 $\mathrm{BOEC}$, we determined the localization of proteins that are subject to AP-3 dependent trafficking. Both the tetraspanin CD63 and the leukocyte receptor CD62P continuously cycle between plasma membrane and WPB., However, after endocytic retrieval, CD63 is incorporated in maturing WPB through transfer from AP-3 positive endosomes, while P-selectin diverges to the trans-Golgi network where it is incorporated in nascent WPB.? Therefore, we stained HPS-2 and healthy control BOEC for vWF and CD63 or P-selectin. We observed that CD63 is not detectable in WPB in HPS-2 BOEC and can only be observed in round endosome-like structures (Figure 1E, left). Quantitative evaluation of our imaging data showed that in WT BOEC, nearly a third of cellular CD63 is associated with WPB (Figure 1 right top), and that on average about $60 \%$ of the WPB are CD63 positive (1E right bottom), numbers that are both in close accordance with previous studies. ${ }^{7,22}$ We observed a sharp reduction in these parameters in HPS-2 BOEC pointing to a defect in CD63 trafficking. P-selectin trafficking to WPB is unaffected by the lack of AP-3 $\beta 1$ (Online Supplementary Figure S1A). We also measured CD63 surface expression in HPS-2 and healthy control BOEC under steady state conditions using flow cytometry. We observed that CD63 surface levels were significantly increased in HPS-2 BOEC (Online
Supplementary Figure $S 1 B)$. Taken together these data point to defective AP-3 dependent trafficking mechanisms in HPS-2 BOEC.

To further corroborate the role of AP-3 in trafficking of CD63, we also generated AP3B1 knock-out primary endothelial cells using CRISPR-Cas9 gene editing. Using lentiviral transduction of cord blood BOEC (cbBOEC), with guide (gRNA) targeting the first exon of the AP3B1 gene, we generated 3 clonal cbBOEC lines containing indels that led to frame shifts and subsequent premature stop codons in the AP3B1 gene (Figure 1C). This resulted in complete abolishment of AP-3 $\beta 1$ expression in all three clonal lines (Figure 1D). Similar to what we observed in HPS-2 BOEC, and to what has been reported in endothelial cells after siRNA-mediated AP-3 $\beta 1$ silencing, ${ }^{7}$ WPB of $A P 3 B 1^{-}$cbBOEC did not contain CD63 (Figure 1F and Online Supplementary Figure S2).

\section{AP3B1-deficient HPS-2 blood outgrowth endothelial cells have an unstable AP-3 complex and lack the Weibel-Palade body v-SNARE VAMP8}

To better understand the phenotypic effect of loss of AP-3 $\beta 1$ expression, we performed a comparative analysis of the whole proteome between HPS-2 and healthy control BOEC by means of label-free LC-MS/MS. We identified 5812 proteins, from which 4323 were quantifiable following the selection criteria. To include the natural variation in our analysis, we compared HPS-2 BOEC to four independent healthy control donors. Z-scored LFO values of the proteins with the highest variation between samples $(\mathrm{S} 0=0.4, \mathrm{FDR}=0.05)$ are shown in the heatmap in Figure 2A. Following this approach, we were able to confirm the AP-3 $\beta 1$ depletion in HPS-2 BOEC (Figure 2B). Moreover, we observed that the expression of AP-3 $\mu 1$, an AP-3 subunit encoded by AP3M1, was also significantly reduced. However, expression levels of AP$3 \delta 1$ and AP-3 o1, the other two subunits of the AP-3 complex, were not significantly different and only modestly reduced (Figure $2 \mathrm{~B}$ ). A similar observation has previously been made in HPS-2 fibroblasts ${ }^{23}$ and in murine pe fibroblasts (pearl, pe, mouse model of HPS-2), where $\delta 1$ and $\sigma 1$ subunits remained as a heterodimer and showed cytosolic rather than membrane-associated localization. ${ }^{24}$ The tight correlation of $\beta 1$ and $\mu 1$ expression levels suggests that loss of AP-3 $\beta 1$ and consequential disintegration of the AP- 3 complex destabilizes the AP- $3 \mu 1$ subunit. This was supported by the observation that lentivirally expressed mEGFP-AP-3 $\beta 1$ was able to rescue the expression of AP-3 $\mu 1$ in HPS-2 BOEC (Figure 2C).

Interestingly, among the down-regulated proteins, we discovered that the expression of the WPB-localized SNARE protein VAMP8 is severely diminished (Figure 2A and Online Supplementary Figure S3). This was specific for VAMP8 as the expression levels of other SNARE proteins that have been implicated in WPB exocytosis were not significantly altered (Online Supplementary Figure S3). Western blot analysis also revealed a complete depletion of VAMP8 expression in HPS-2 BOEC compared to healthy controls (Figure 2D). Moreover, immunofluorescent staining in healthy control and HPS-2 BOEC for VAMP8 and vWF, showed that WPB in HPS-2 BOEC lack VAMP8 immunoreactivity (Figure 2E). In addition, in the AP3B1 knockout $\mathrm{BOEC}$ lines, VAMP8 was strongly reduced when compared to the control cbBOEC (Online Supplementary Figure S4). 
Ex vivo HPS-2 endothelial cells have impaired stimulus-induced Weibel-Palade body exocytosis

To investigate the contribution of AP-3 dependent maturation on the degranulation efficiency of WPB in endothelial cells, we measured the stimulus-induced vWF release in HPS-2 BOEC. We observed that, under unstimulated conditions, the release of vWF is unaffected in the HPS-2 BOEC compared to WT (Figure 3A). However, upon stimulation, HPS-2 BOEC showed a clear defect in vWF release with both $\mathrm{Ca}_{2}^{+}$(histamine) and cAMP-mediated (forskolin) secretagogues (Figure 3B). As an alternative measurement of WPB exocytosis, we also investigated how other WPB proteins respond to histamine treatment. We stimulated WT and HPS-2 BOEC and measured CD62P exposure on the plasma membrane (Figure 3D). We found that significantly less CD62P was expressed on HPS2 BOEC plasma membrane when compared to healthy control BOEC upon histamine stimulation. The reduction in the stimulus-induced surface expression of CD62P and release of vWF in HPS-2 BOEC suggests that defects in the AP-3 dependent maturation of WPB alter the exocytotic potential of these organelles in endothelial cells.
A

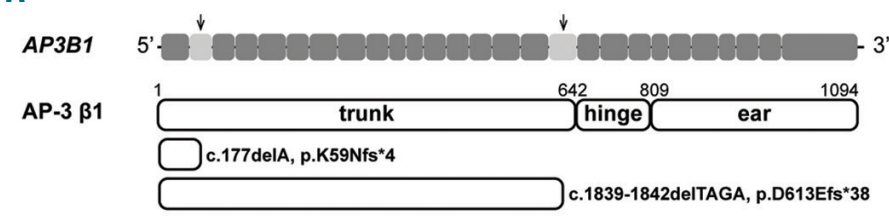

C

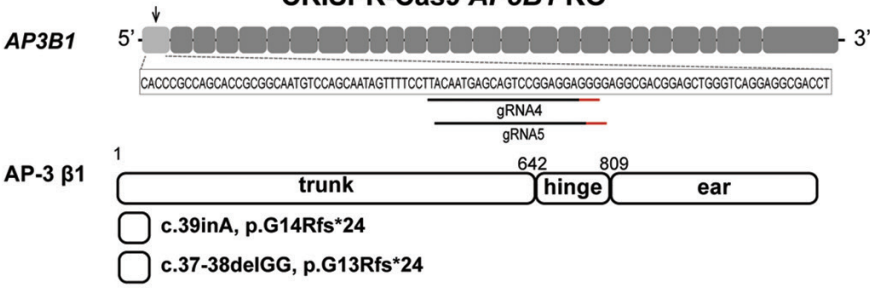

B

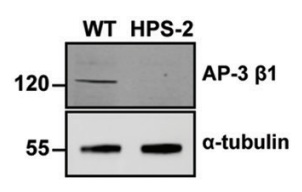

D

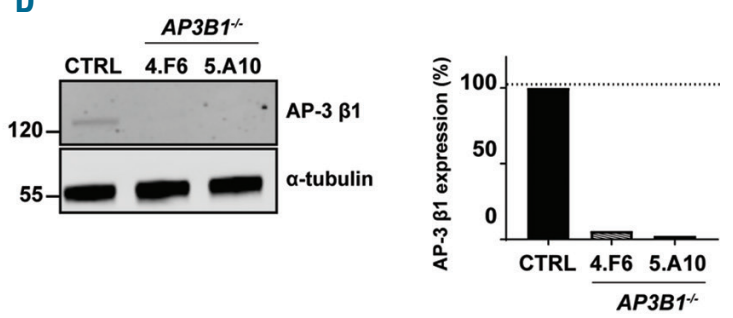

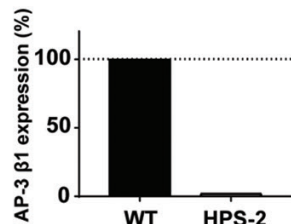

WT HPS-2
E

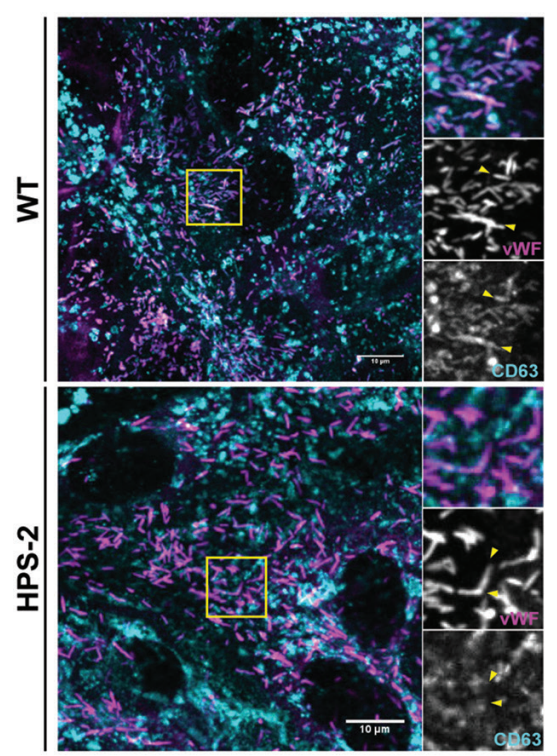

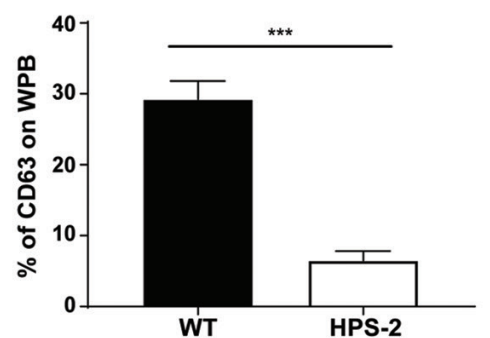

F

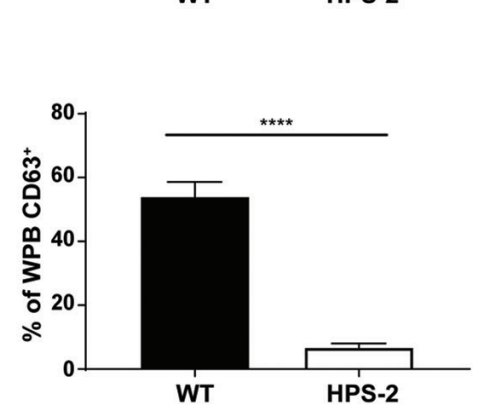

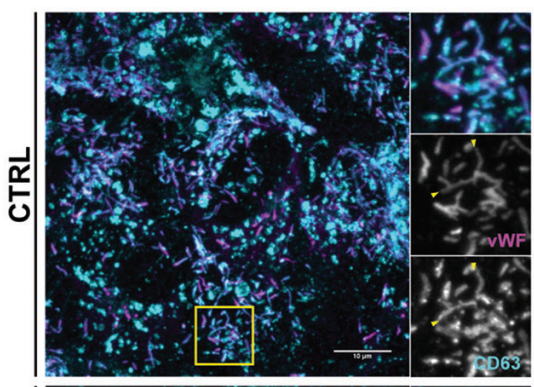

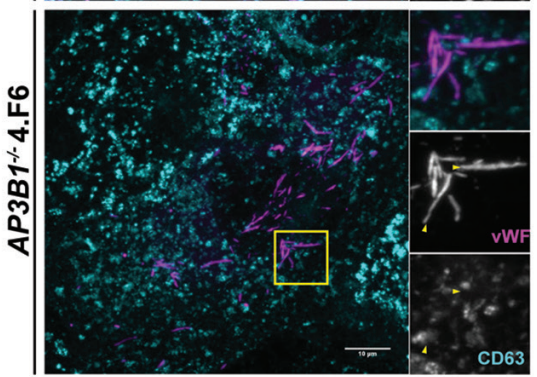

Figure 1. Disrupted trafficking of CD63 to Weibel-Palade bodies in ex vivo patient-derived HPS-2 BOEC and CRISPR/Cas9-engineered AP3B1 ${ }^{/ /}$BOEC. (A) Cartoon depicting the mutations of the HPS-2 patient in the AP3B1 gene and the corresponding predicted truncated protein products relative to the full length AP-3 31 domain structure. (B) Expression of AP-3 $\beta 1$ in WT and HPS-2 BOEC. (B) Healthy WT and HPS-2 BOEC lysates were separated with SDS-PAGE and were immunoblotted for AP$3 \beta 1 ; \alpha$-tubulin was used as a loading control. Molecular weight standards are indicated on the left (kDa) and quantification of relative AP-3 $\beta 1$ expression in HPS2 BOEC normalized to AP-3ß1 in healthy WT BOEC (right). (C) Cartoon representation of the CRISPR $\backslash$ Cas9-engineering strategy to generate AP3B1 ${ }^{-/}$BOEC lines. Guide RNA (gRNA4 and gRNA5) are shown underneath a fragment of AP3B1 exon 1 sequence with their protospacer adjacent motif (PAM) indicated in red. The mutations and the corresponding predicted truncated protein products of $2 \mathrm{AP} 3 \mathrm{~B} 1^{-/}$clones (4.F6 and 5.A10, respectively) are shown relative to the full length AP-3 1 domain structure. (D) Loss of AP-3ß1 expression in AP3B1 $1^{-/-}$BOEC. (D) Lysates of clonal CTRL BOEC and 2 clonal AP3B1 $1^{-/-}$BOEC lines (4.F6 and 5.A10) were separated with SDS-PAGE and were immunoblotted for AP-3 $\beta 1$; $\alpha$-tubulin was used as a loading control (left) and quantification of relative AP-3 $\beta 1$ expression in clonal AP3B1 ${ }^{-1-}$ BOEC lines normalized to AP-3ß1 in clonal CTRL BOEC (right). (E-F) Immunostaining of BOEC for vWF (magenta) and CD63 (cyan) in WT versus HPS-2 BOEC (E left) and CTRL versus AP3B1 ${ }^{-1-}$ 4.F6 BOEC (F), respectively. Boxed areas are magnified in the right part. Yellow arrowheads indicate the position of WPB in both channels. Scale bars represent $10 \mu \mathrm{m}$. (E) Proportion of CD63 immunoreactivity that is found on WPB (right, top) and proportion of WPB that contain CD63 immunoreactivity (right, bottom). Student $t$-test, $* * * P<0.001, * * * * P<0.0001$. 
Weibel-Palade bodies v-SNARE protein VAMP8 promotes Weibel-Palade body exocytosis

Expression of the SNARE protein VAMP8 is reduced in the absence of AP-3 $\beta 1$, in HPS-2 patient BOEC as well as in CRISPR-Cas9 engineered AP3B1 knockout endothelial cells. SNARE proteins are key regulators of intracellular membrane fusion events, such as during exocytosis or during fusion between organelles. To further investigate the role of VAMP8 in endothelial cells, we first studied the intracellular localization of VAMP8 with confocal microscopy in HUVEC/healthy control BOEC. In line with previous reports, VAMP8 was localized on a subset of WPB and on spherical organelles of the endosomal system (Online Supplementary Figure S5). ${ }^{21,25}$ Interestingly, VAMP8-positive WPB and VAMP8-positive endosomes both contained CD63, suggesting their shared itinerary may be indicative of a common AP-3 dependent trafficking pathway.

To explore the role of VAMP8 in endothelial cells we generated clonal CRISPR-Cas9 knockout BOEC by introducing a mutation in the first and second exon (Figure 4A) and evaluated the knockout efficiency by western blot (Figure 4B) and immunofluorescence (Online Supplementary Figure S6A). We investigated both AP-3-dependent intracel-
A
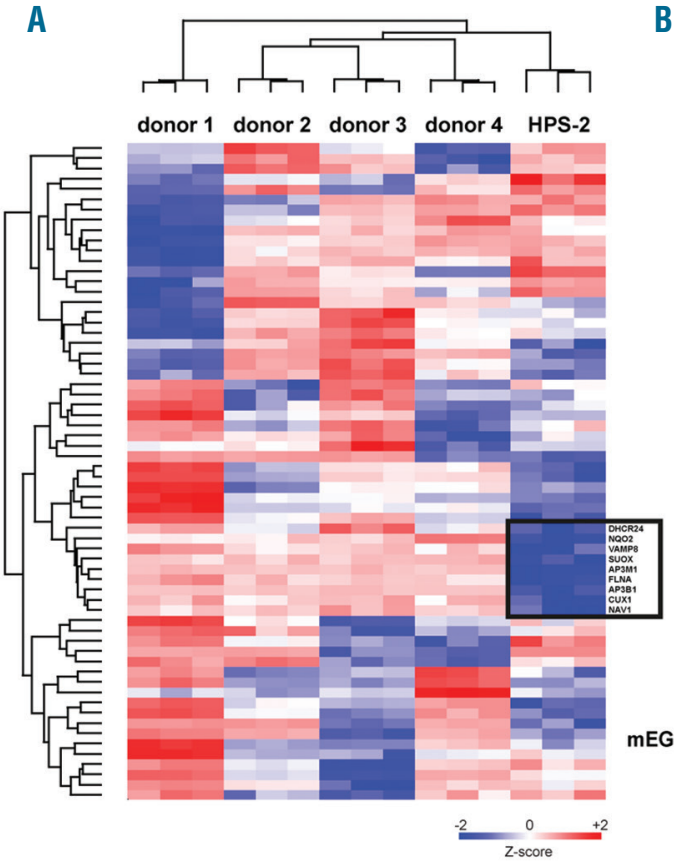

E

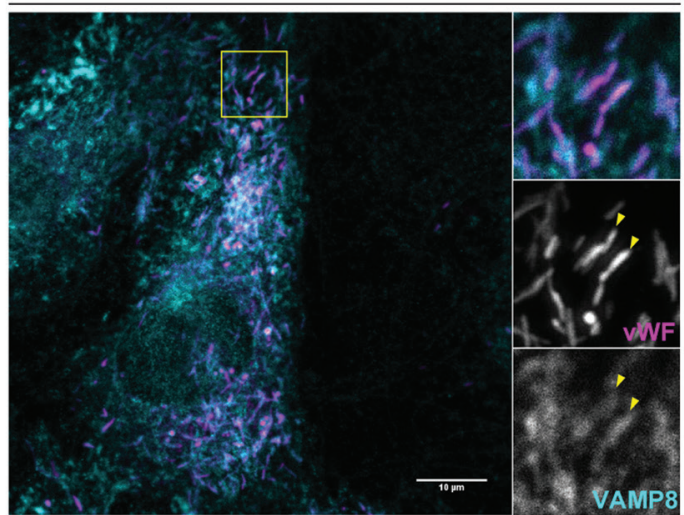

B
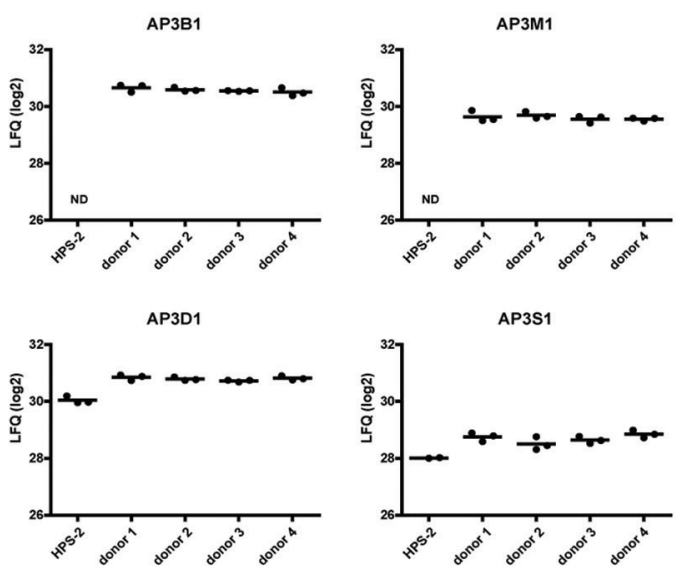

C

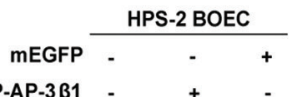

D
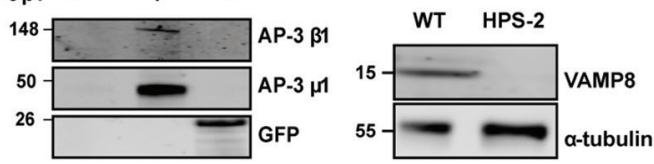

\section{WT}

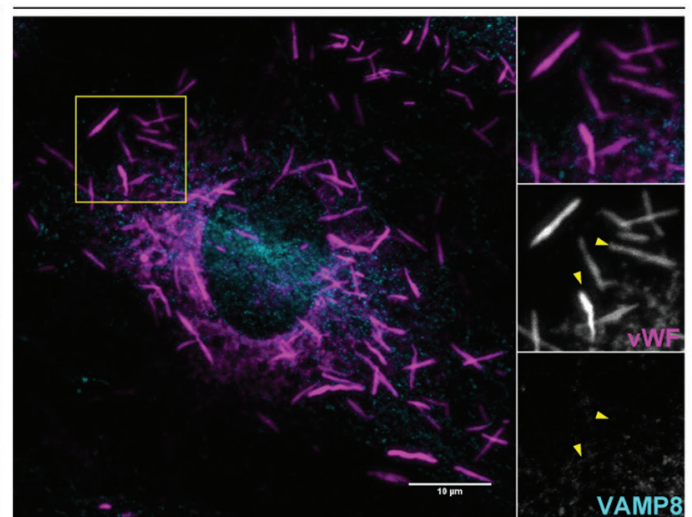

Figure 2. HPS-2 BOEC lack components of the AP-3 complex and the WPB v-SNARE VAMP8. (A and B) Whole proteome analysis of HPS-2 BOEC. (A) Heatmap of Zscored LFQ $\left(\log _{2}\right)$ values of the proteins with the highest variation between BOEC derived from 4 individual healthy donors and HPS2 derived BOEC (ANOVA SO=0.4 FDR $=0.05)(B)$ Graph representing LFQ $\left(\log _{2}\right)$ values for AP3B1, AP3D1, AP3M1 and AP3S1. (C) HPS-2 BOEC lentivirally transduced with mEGFP-AP-3 31 and mEGFP (control). Lysates were separated with SDS-PAGE and were immunoblotted for AP-3 $\beta 1$, AP-3 $\mu 1$ and GFP; molecular weight standards are indicated on the left (kDa). (D) Immunoblot analysis of VAMP8 in lysates of HPS-2 and WT BOEC; $\alpha$-tubulin was used as a loading control.(E) Immunofluorescent stainings for von Willebrand factor (magenta) and VAMP8 (cyan) in healthy WT and HPS-2 BOEC. Boxed areas are magnified in the right part. Yellow arrowheads indicate the position of WPB in both channels. Scale bars represent $10 \mu \mathrm{m}$. 
lular protein trafficking as well as the efficiency of WPB to exocytose upon stimulation in two clonal VAMP8 ${ }^{-/-}$BOEC lines. To assess the involvement of VAMP8 in a fusion step between WPB and the endosomal compartment during CD63 recruitment, we checked the localization of CD63 in control and VAMP8-deficient cell lines. Immunofluorescent staining of CD63 and vWF in the VAMP8 ${ }^{-/}$lines exhibit a similar pattern when compared to the control cell lines, with CD63 being found on endosome-like structures as well as WPB (Online Supplementary Figure $S 6 B$ ). This suggests that membrane transfer between endosomes and WPB during CD63 trafficking does not depend on VAMP8. We next examined the involvement of VAMP8 in WPB exocytosis by testing stimulus-induced vWF release in CRISPR-edited VAMP8 $8^{-/}$BOEC. Our data show that the intracellular levels of $\mathrm{vWF}$ are similar for VAMP8-deficient and control cell lines (Figure 4C), showing that the process of CRISPR-Cas9 genetic modification and clonal selection does not affect vWF biosynthesis and/or WPB biogenesis. However, upon $\mathrm{Ca}_{2}^{+}$-mediated stimulation of WPB release with histamine, VAMP8 knockout endothelial cells secreted significantly less vWF when compared to the control lines (Figure 4D). These findings demonstrate that VAMP8 promotes stimulus-induced vWF secretion and establish VAMP8 as a novel component of the WPB exocytotic machinery.

\section{Discussion}

The AP-3 complex regulates the formation and maturation of lysosome-related organelles in many different cell types. ${ }^{8}$ In this study, we show that mutations in the $A P 3 B 1$ gene that lead to HPS-2 result in loss of AP-3 $\beta 1$ and rapid degradation of the AP-3 $\mu 1$ subunit of the AP-3 complex. While the $\delta 1$ and $\sigma 1$ subunits are still expressed, the consequential destabilization of the AP-3 complex leads to a failure to traffic proteins to the WPB. Two of these, CD63 and VAMP8, normally co-reside on endosomes and WPB. However, in the absence of AP-3-dependent sorting, their fates differ radically. Blockade of the route from the endosomal compartment to the WPB causes an increase of CD63 on the cell surface, possibly caused by a global redistribution of CD63 in the absence of its storage compartment. This has previously been reported for other cell types derived from HPS-2 patients, such as cytotoxic T lymphocytes and fibroblasts. ${ }^{13-15,23}$ VAMP8 expression is severely reduced in both HPS-2 BOEC and in CRISPRengineered AP3B1 KO BOEC, for which we currently do not have an explanation. Contrary to CD63, VAMP8 does not contain any of the known sorting motifs that would allow it to directly interact with the AP-3 complex., ${ }^{9,10}$ VAMP7, a v-SNARE that is found on a number of LRO, has been shown to interact with the AP-3 complex on
A

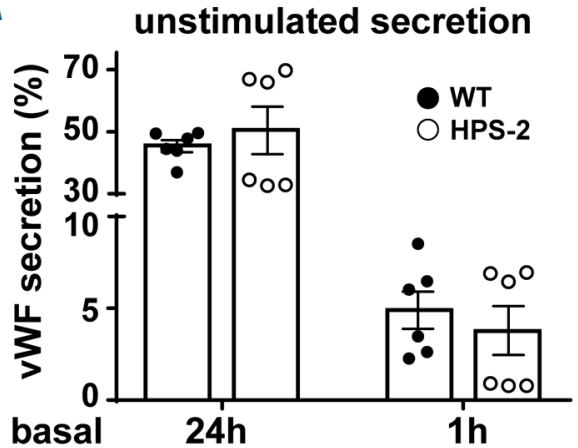

B

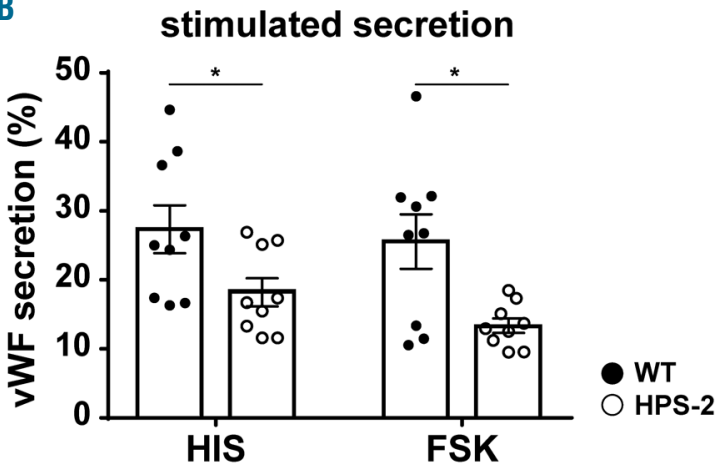

C

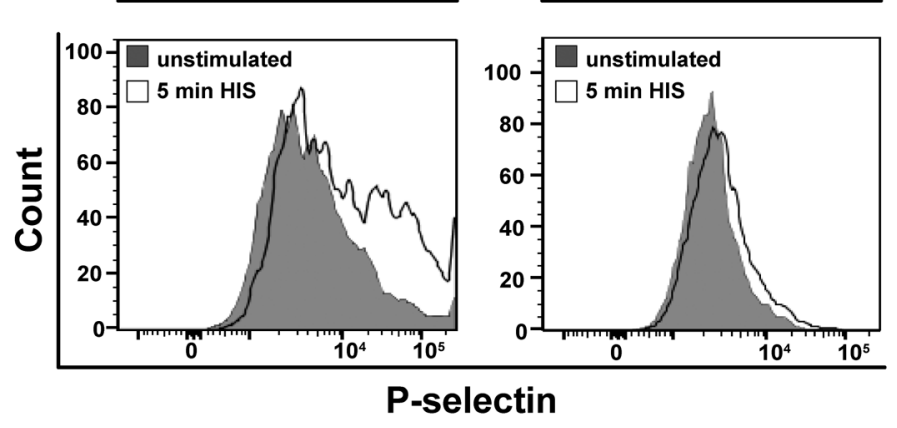

D CD62P exposure

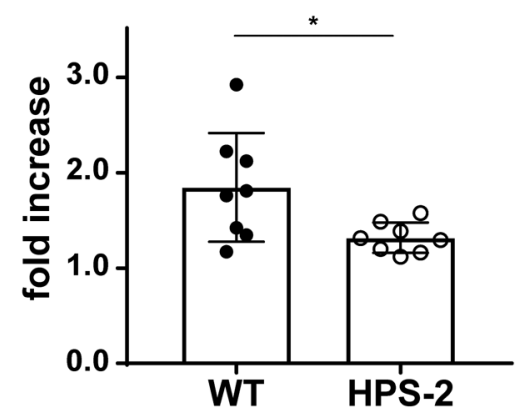

Figure 3. Impaired stimulus-induced von Willebrand Factor (VWF) secretion and P-selectin cell surface exposure in HPS-2 blood outgrowth endothelial cells (BOEC). (A) Basal (unstimulated) secretion of vWF in conditioned media after 24 hours and 1 hour from WT HPS-2 (black) and HPS-2 (white) BOEC ( $n=6$ ). (B) Stimulated vWF secretion after 30 minutes treatment with $100 \mu \mathrm{M}$ histamine (HIS) or $10 \mu \mathrm{M}$ forskolin $+100 \mu \mathrm{M}$ IBMX (FSK) from WT (black) and HPS-2 (white) BOEC. Secretion is expressed as relative proportion of intracellular vWF in unstimulated cells $(n=9)$. (C) Representative histograms of P-selectin (CD62P) cell surface expression before (close gray) and after 5 minutes stimulation with $100 \mu \mathrm{M}$ histamine (open black line) (D) CD62P exposure after histamine stimulation expressed as fold increase over unstimulated cells $(\mathrm{n}=8)$. Two tailed Student $t$-test, $* P<0.05$. 
early endosomes via a direct interaction between the AP-3 $\delta 1$ subunit and the $\mathrm{N}$-terminal longin domain of VAMP7. ${ }^{26,27}$ Destabilization of the AP-3 complex, such as in mocha mice which lack the $\delta 1$ subunit of AP-3, leads to mistargeting of VAMP7. ${ }^{27}$ It has also been suggested that AP-3 indirectly traffics STX13 to maturing melanosomes via VAMP7. ${ }^{28}$ Although VAMP8 does not contain a longin domain, it may be possible that during WPB maturation there is a similar mechanism by which VAMP8 latches on to the back of another AP-3 interacting partner.

In this study, we further explored the effect of WPB maturation on regulated secretion and identify VAMP8 as a novel component of the exocytotic fusion machinery. Previous studies have found that WPB contain the VSNAREs VAMP3 and VAMP8, but so far only VAMP3 has been implicated in $\mathrm{Ca}^{2}$-dependent WPB exocytosis. ${ }^{25} \mathrm{We}$ had previously established that VAMP8 is a direct interactor of both syntaxin-3 (STX3) and syntaxin-4 (STX4). ${ }^{21}$ STX4, which is found at the plasma membrane, has been implicated in thrombin-induced release of WPB through the formation of a complex with WPB-localized
VAMP3 ${ }^{25,29}$ We have recently shown that STX3 is localized on WPB and that absence of STX3 from endothelial cells results in impaired basal and hormone-evoked vWF secretion. ${ }^{21}$ The decrease in histamine-evoked vWF secretion in VAMP8 knock-out endothelial cells indicates an active involvement of VAMP8 in stimulus-induced WPB exocytosis. Based on its ability to interact with STX4 and STX3, VAMP8 can potentially support exocytosis via direct (WPB-plasma membrane) or homotypic (WPBWPB) fusion modes. Further research on this topic should address the involvement of VAMP8 in the different modes of fusion.

In vivo, VAMP8 $\%$ mice show delayed and decreased thrombus formation. ${ }^{30}$ Although, in their study, Graham et al. found that defective thrombus formation correlated with impaired dense granule release from $V A M P 8^{-1}$ platelets, they did not test whether lack of VAMP8 in endothelial cells also contributed to defects in thrombus formation. A more pronounced defect in thrombus formation was also observed in ruby eye mice, a murine model for HPS-6, which lack platelet dense granules. $^{31}$
A

\section{CRISPR-Cas9 VAMP8 KO}

VAMP8

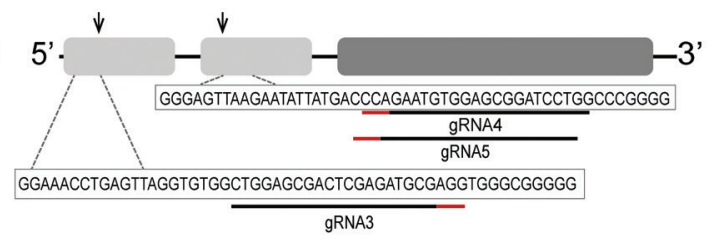

B

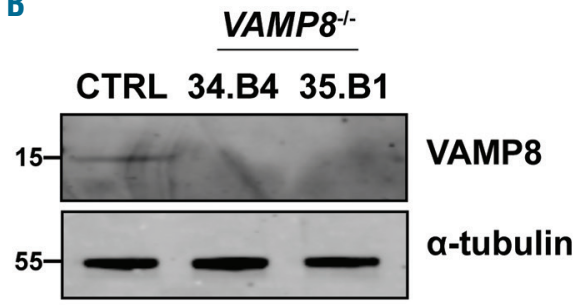

C intracellular vWF

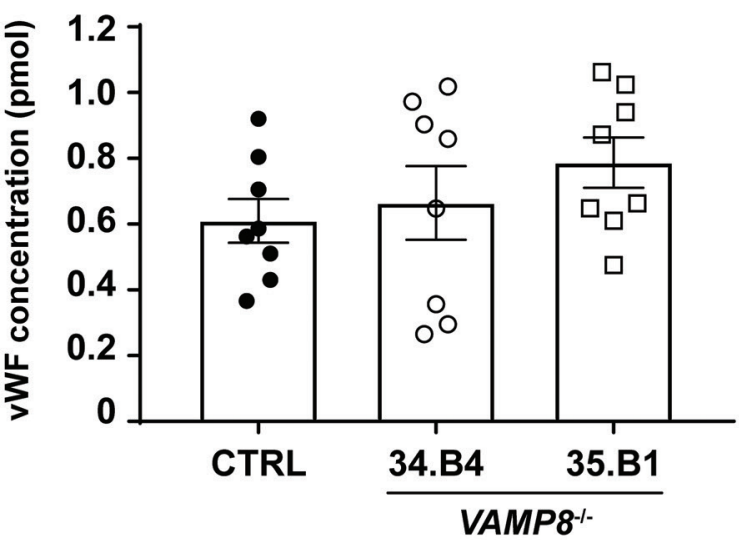

D stimulated secretion

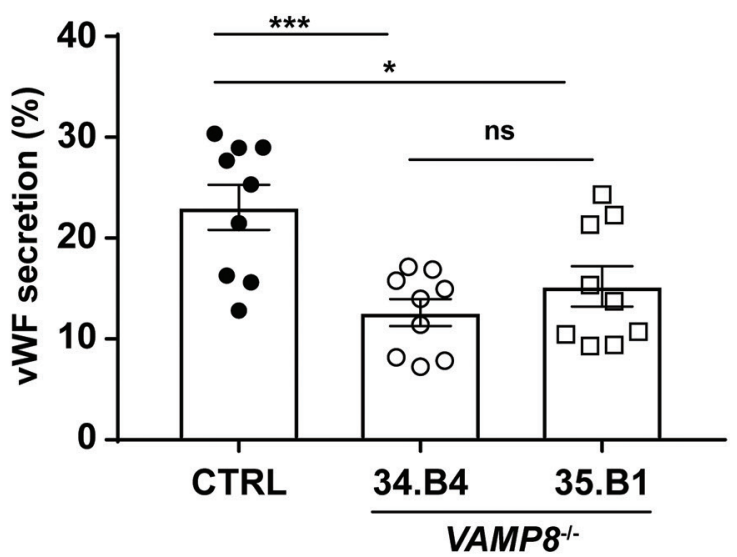

Figure 4. CRISPR-Cas9-engineered VAMP8 blood outgrowth endothelial cells (BOEC) have decreased histamine-induced von Willebrand Factor (vWF) secretion. A and B) Generation of clonal VAMP8 deficient BOEC lines using CRISPR/Cas9 genome-editing. (A) Cartoon representation of the CRISPR/Cas9-engineering strategy to generate VAMP8 ${ }^{--}$BOEC lines. Guide RNA targeting exon 1 and exon 2 (gRNA3 and gRNA4\&5, respectively) are shown underneath fragments of VAMP8 exon 1 and 2 sequences with their protospacer adjacent motif (PAM) indicated in red. (B) Loss of VAMP8 expression in clonal VAMP8 ${ }^{-1-}$ BOEC. Lysates of clonal CTRL BOEC and 2 clonal VAMP8 BOEC lines (34.B4 and 35.B1) were separated with SDS-PAGE and were immunoblotted for VAMP8; $\alpha$-tubulin was used as a loading control. Molecular weight standards are indicated on the left (kDa). (C) Intracellular levels of vWF in lysates from control and 2 clonal VAMP8 $\%$ KO BOEC lines (34.B4 and 35.B1) as determined by ELISA ( $n=9$ ). (D) Stimulated VWF secretion after 30 minutes treatment with $100 \mu \mathrm{M}$ histamine (HIS) from CTRL (close black) and 2 clonal VAMP $^{-/-}$KO BOEC lines (34.B4 and 35.B1) (open circle and square respectively). Secretion of vWF in supernatant from control and 2 clonal VAMP8 ${ }^{-1-}$ KO BOEC lines (34.B4 and 35.B1). Secretion is expressed as relative proportion of intracellular vWF in unstimulated cells $(n=9)$. Two tailed Student $t$-test, ns: $P>0.05$; $* P<0.05$; $* * * P<0.001$. 


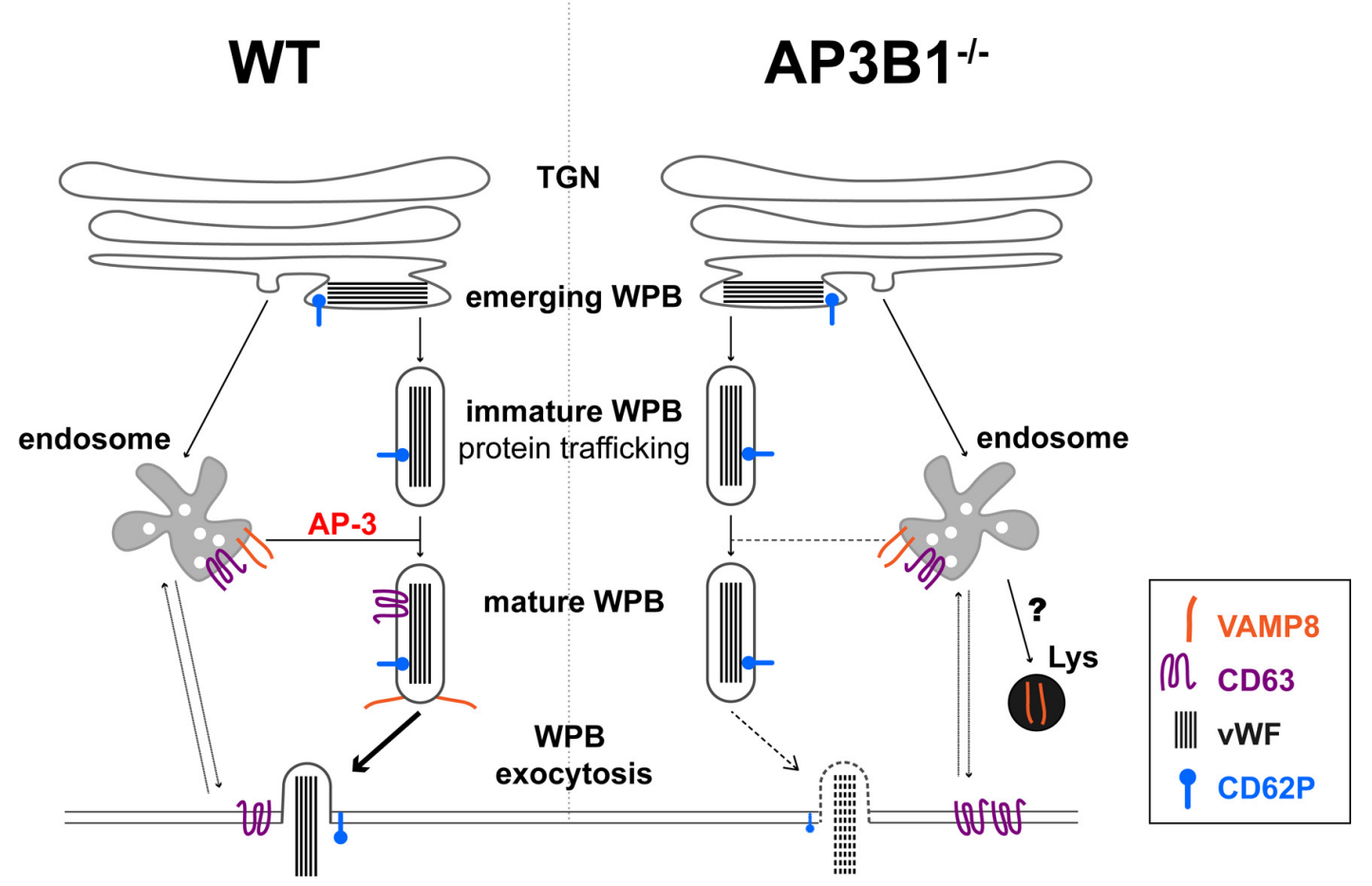

Figure 5. Proposed model of (defective) AP-3 dependent acquisition of VAMP8 during Weibel-Palade bodies (WPB) maturation. WPB in normal endothelial cells (WT, left) emerge from the trans-Golgi network (TGN) and mature by acquisition of recycled membrane proteins, such as CD63 and VAMP8, through endosomal trafficking to the WPB membrane via an AP-3 dependent pathway. Recruitment of VAMP8 promotes the secretion competence of WPB by supporting the formation of trans-SNARE complexes with STX3 and STX4, ${ }^{21}$ which will allow WPB to undergo exocytosis upon cellular activation. After membrane fusion, CD63 and VAMP8 are retrieved and, via the early endosome (EE), make their way to another WPB. In the absence of a function AP-3 complex (right), such as in HPS-2 or AP3B1\% BOEC, WPB fail to recruit CD63 from the early endosomes. This leads to a global redistribution of cellular CD63, increasing its presence on the cell surface during steady state conditions. WPB also fail to acquire (part of) their fusion machinery such as VAMP8, resulting in attenuated WPB exocytosis in response to cellular activation. Following its failure to transfer to the WPB membrane, VAMP8 is possibly rerouted to the lysosome where it is degraded.

Contributing to this phenotype was: (i) the inability to secrete protein disulfide isomerase (PDI) from so called Tgranules, a tubular secretory compartment in platelets that contains VAMP8; ${ }^{32}$ and (ii) defective thrombin-induced secretion of PDI and VWF from endothelial cells after silencing of HPS6. ${ }^{31}$ A recent study also found that endothelial secretion of vWF in response to administration of the vasopressin analog 1-deamino-8-D-arginine vasopressin (DDAVP) was delayed and/or decreased in pallid, ruby eye and pale ear mice, murine models of HPS-9, HPS6 and HPS-1, respectively. ${ }^{33}$ This provides an explanation for earlier observations that long bleeding times in ruby eye and pale ear mice were not corrected by DDAVP, ${ }^{34}$ but possibly also for the variable response of HPS patients to DDAVP. ${ }^{35-37}$ Taken together, this suggests that defects in maturation of WPB decrease their ability to undergo exocytosis.

In this study, we show that BOEC from an HPS-2 patient have a moderate (but not complete) defect in stimulated vWF secretion, a phenotype that we found to be closely correlated with the absence of VAMP8 on mature WPB. We propose that, during maturation, LRO also acquire components of their fusion machinery, thereby increasing their ability to undergo exocytosis. In some LRO, such as dense granules, defects in LRO maturation result in the absence of the secretory granule at issue, which obscures a secretory defect by the lack of the fusion machinery recruitment. This becomes apparent in LRO, such as WPB, which have already been formed before their failure to interact with the endo/lysosomal system, preventing them from acquiring additional content (CD63) or fusion machinery components (VAMP8) (Figure 5).

\section{Funding}

This study was supported by grants from the Landsteiner Stichting voor Bloedtransfusie Research (LSBR-1244, LSBR1517 and LSBR-1707), Sanquin (PPOC-2015-24P and PPOC-2018-21) and the Dutch Thrombosis Foundation (TSN 56-2015 and 2017-01). RB is supported by a European Hematology Association Research Fellowship.

\section{References}

1. Schillemans $M$, Karampini E, Kat $M$, Bierings R. Exocytosis of Weibel-Palade bodies: how to unpack a vascular emergency kit. J Thromb Haemost. 2019;17(1):6-18.
2. Metcalf DJ, Nightingale TD, Zenner HL, LuiRoberts WW, Cutler DF. Formation and function of Weibel-Palade bodies. J Cell Sci. 2008;31(9):882-888.

3. Rondaij MG, Bierings $\mathrm{R}$, Kragt A, van Mourik JA, Voorberg J. Dynamics and plas- ticity of Weibel-Palade bodies in endothelial cells. Arterioscler Thromb Vasc Biol 2006;26(5):1002-1007.

4. Marks MS, Heijnen HFG, Raposo G. Lysosome-related organelles: Unusual compartments become mainstream. Curr Opin 
Cell Biol. 2013;25(4):495-505.

5. Kobayashi T, Vischer UM, Rosnoblet C, et al. The tetraspanin CD63/lamp3 cycles between endocytic and secretory compartments in human endothelial cells. Mol Biol Cell. 2000;11(5):1829-1843.

6. Arribas M, Cutler DF. Weibel-Palade body membrane proteins exhibit differential trafficking after exocytosis in endothelial cells. Traffic. 2000;1(10):783-793.

7. Harrison-Lavoie KJ, Michaux G, Hewlett L, et al. P-selectin and CD63 use different mechanisms for delivery to Weibel-Palade bodies. Traffic. 2006;7(6):647-662.

8. Robinson MS, Bonifacino JS. Adaptor-related proteins. Curr Opin Cell Biol. 2001;13(4):444-453.

9. Bonifacino JS, Traub LM. Signals for sorting of transmembrane proteins to endosomes and lysosomes. Annu Rev Biochem. 2003;72:395-72447.

10. Newell-Litwa K, Seong E, Burmeister M, Faundez V. Neuronal and non-neuronal functions of the AP-3 sorting machinery. J Cell Sci. 2007;120(Pt 4):531-541.

11. Rous BA, Reaves BJ, Ihrke G, et al. Role of adaptor complex AP-3 in targeting wild-type and mutated CD63 to lysosomes. Mol Biol Cell. 2002;13(3):1071-1082.

12. Wei ML. Hermansky-Pudlak syndrome: a disease of protein trafficking and organelle function. Pigment Cell Res. 2006;19(1):1942.

13. Dell'Angelica EC, Shotelersuk V, Aguilar RC, Gahl WA, Bonifacino JS. Altered trafficking of lysosomal proteins in HermanskyPudlak syndrome due to mutations in the beta $3 \mathrm{~A}$ subunit of the AP-3 adaptor. Mol Cell. 1999:3(1):11-21.

14. Clark RH, Stinchcombe JC, Day A, et al. Adaptor protein 3-dependent microtubulemediated movement of lytic granules to the immunological synapse. Nat Immunol. 2003;4(11):1111-1120.

15. Wenham M, Grieve S, Cummins $M$, et al. Two patients with Hermansky Pudlak syndrome type 2 and novel mutations in AP3B1. Haematologica. 2010;95(2):333-337.

16. de Boer M, van Leeuwen K, Geissler J, et al. Hermansky-Pudlak syndrome type 2: Aberrant pre-mRNA splicing and mislocalization of granule proteins in neutrophils. Hum Mutat. 2017;38(10):1402-1411.

17. Martin-Ramirez J, Kok MGM, Hofman M, et al. Individual with subclinical atherosclerosis have impaired proliferation of blood outgrowth endothelial cells, which can be restored by statin therapy. PLoS One. 2014;9(6):e99890.

18. Bierings R, Hellen N, Kiskin N, et al. The interplay between the Rab27A effectors Slp4-a and MyRIP controls hormone-evoked Weibel-Palade body exocytosis. Blood 2012;120(13):2757-2767.

19. van Breevoort D, Snijders AP, Hellen N, et al. STXBP1 promotes Weibel-Palade body exocytosis through its interaction with the Rab27A effector Slp4-a. Blood. 2014;123 (20):3185-3194

20. Azevedo C, Burton A, Ruiz-Mateos E, Marsh M, Saiardi A. Inositol pyrophosphate mediated pyrophosphorylation of AP3B1 regulates HIV-1 Gag release. Proc Natl Acad Sci U S A. 2009;106(50):21161-21166.

21. Schillemans M, Karampini E, van den Eshof $\mathrm{BL}$, et al. Weibel-Palade Body Localized Syntaxin-3 Modulates Von Willebrand Factor Secretion From Endothelial Cells. Arterioscler Thromb Vasc Biol. 2018;38(7): 1549-1561.

22. Poeter M, Brandherm I, Rossaint I, et al Annexin A8 controls leukocyte recruitment to activated endothelial cells via cell surface delivery of CD63. Nat Commun. 2014;5 (3738):3738.

23. Liu L, Sutton J, Woodruff E, Villalta F Spearman P, Dong X. Defective HIV-1 particle assembly in AP-3-deficient cells derived from patients with Hermansky-Pudlak syndrome type 2. J Virol. 2012;86(20):1124211253.

24. Peden AA, Rudge RE, Lui WWY, Robinson MS. Assembly and function of AP-3 complexes in cells expressing mutant subunits. J Cell Biol. 2002;156(2):327-336.

25. Pulido IR, Jahn R, Gerke V. VAMP3 is associated with endothelial Weibel-Palade bodies and participates in their $\mathrm{Ca}(2+)$-dependent exocytosis. Biochim Biophys Acta. 2011;1813(5):1038-1044.

26. Martinez-Arca S, Rudge R, Vacca M, et al. A dual mechanism controlling the localization and function of exocytic v-SNAREs. Proc Natl Acad Sci U S A. 2003;100(15):90119006.

27. Kent HM, Evans PR, Schäfer IB, et al. Structural basis of the intracellular sorting of the SNARE VAMP7 by the AP3 adaptor complex. Dev Cell. 2012;22(5):979-988.

28. Jani RA, Purushothaman LK, Rani S, Bergam P, Setty SRG. STX13 regulates cargo delivery from recycling endosomes during melanosome biogenesis. J Cell Sci. 2015;128(17):3263-3276.

29. Fu J, Naren AP, Gao X, Ahmmed GU, Malik AB. Protease-activated receptor- 1 activation of endothelial cells induces protein kinase Calpha-dependent phosphorylation of syntaxin 4 and Munc18c: role in signaling $p$ selectin expression. J Biol Chem. 2005;280 (5):3178-3184.

30. Graham GJ, Ren $Q$, Dilks JR, Blair $P$ Whiteheart SW, Flaumenhaft R Endobrevin/VAMP-8-dependent dense granule release mediates thrombus formation in vivo. Blood. 2009;114(5):1083-1090.

31. Sharda A, Kim SH, Jasuja R, et al. Defective PDI release from platelets and endothelial cells impairs thrombus formation in Hermansky-Pudlak syndrome. Blood. 2015;125(10):1633-1642.

32. Thon JN, Peters CG, Machlus KR, et al. T granules in human platelets function in TLR9 organization and signaling. J Cell Biol. 2012;198(4):561-574.

33. Ma J, Zhang Z, Yang L, Kriston-Vizi J, Cutler DF, Li W. BLOC-2 subunit HPS6 deficiency affects the tubulation and secretion of von Willebrand factor from mouse endothelial cells. J Genet Genomics. 2016;43(12):686693.

34. Sweeney JD, Novak EK, Reddington M Takeuchi KH, Swank RT. The RIIIS/J inbred mouse strain as a model for von Willebrand disease. Blood. 1990;76(11):2258-2265.

35. Van Dorp DB, Wijermans PW, Meire F, Vrensen G. The Hermansky-Pudlak syndrome. Variable reaction to 1-desamino-8Darginine vasopressin for correction of the bleeding time. Ophthalmic Paediatr Genet. 1990;11(3):237-244.

36. Zatik J, Póka R, Borsos A, Pfliegler G. Variable response of Hermansky-Pudlak syndrome to prophylactic administration of 1-desamino 8D-arginine in subsequent pregnancies. Eur J Obstet Gynecol Reprod Biol. 2002;104(2):165-166.

37. Cordova A, Barrios NJ, Ortiz I, Rivera E, Cadilla C, Santiago-Borrero PJ. Poor response to desmopressin acetate (DDAVP) in children with Hermansky-Pudlak syndrome. Pediatr Blood Cancer. 2005;44(1):51-54. 